\title{
The impact of JNK inhibitor D-JNKI-I in a murine model of chronic colitis induced by dextran sulfate sodium
}

This article was published in the following Dove Press journal:

Journal of Inflammation Research

2 May 2013

Number of times this article has been viewed

\author{
Sabine Kersting ${ }^{1, *}$ \\ Volker Behrendt ${ }^{1, *}$ \\ Jonas Kersting' \\ Kirstin Reinecke ${ }^{3}$ \\ Christoph Hilgert ${ }^{1}$ \\ Ingo Stricker ${ }^{2}$ \\ Thomas Herdegen ${ }^{3}$ \\ Monika S Janot ${ }^{1}$ \\ Waldemar Uhl' \\ Ansgar M Chromik' \\ 'Department of General and Visceral \\ Surgery, St Josef Hospital, Ruhr \\ University of Bochum, Bochum, \\ Germany; ${ }^{2}$ Department of Pathology, \\ Ruhr University of Bochum, Bochum, \\ Germany; ${ }^{3}$ Institute of Experimental \\ and Clinical Pharmacology, University \\ Hospital of Schleswig-Holstein, Kiel, \\ Germany
}

*The two authors Sabine Kersting and Volker Behrendt contributed equally to this work
Correspondence: Ansgar Chromik Department of General Surgery, Ruhr University of Bochum, St Josef Hospital, Gudrunstrasse 56, 4479I Bochum, Germany Tel +4923450922II $\mathrm{Fax}+492345092209$

Email chromik@t-online.de
Purpose: The c-Jun N-terminal kinases (JNK) are involved in the activation of T cells and the synthesis of proinflammatory cytokines. Several studies have established the relevance of the JNK pathway in inflammatory bowel diseases. The present study analyzed the therapeutic effect of D-JNKI-1, a specific JNK-inhibiting peptide, in a low-dose dextran sulfate sodium (DSS) model of chronic colitis.

Methods: DSS colitis was induced in female C57/BL6 mice by cyclic administration using different concentrations of DSS (1.0\% and $1.5 \%)$. Mice in the intervention groups received subcutaneous administration of $1 \mu \mathrm{g} / \mathrm{kg}$ D-JNKI-1 on days 2,12 , and 22 . They were monitored daily to assess the severity of colitis, body weight, stool consistency, and the occurrence of occult blood or gross rectal bleeding using evaluation of the disease activity index. The animals were sacrificed after 30 days, and the inflamed intestine was histologically evaluated using a crypt damage score. Immunohistochemical quantification of $\mathrm{CD}^{+}$and $\mathrm{CD} 8^{+}$cells was also carried out.

Results: Administration of $1 \mu \mathrm{g} / \mathrm{kg}$ D-JNKI-1 resulted in a significant decrease in the disease activity index ( $P=0.013$ for $1.0 \%$ DSS; $P=0.007$ for $1.5 \% \mathrm{DSS}$ ). As a mild form of colitis was induced, histological examination did not show any distinct damage to the mucosa and crypts. However, expression of $\mathrm{CD}^{+}{ }^{+}$and $\mathrm{CD}^{+}$cells was reduced in mice treated with D-JNKI-1 (not significant).

Conclusion: Administration of D-JNKI-1 resulted in a clinical attenuation of chronic DSS colitis, and a therapeutic effect of D-JNKI-1 must therefore be assumed. The decrease in CD4 ${ }^{+}$ and $\mathrm{CD}^{+}$cells may reflect the influence of D-JNKI-1 on T-cell activation, differentiation, and migration.

Keywords: c-Jun N-terminal kinase inhibitor, dextran sulfate sodium colitis, inflammatory bowel diseases, T cell, D-JNKI-1

\section{Introduction}

Inflammatory bowel diseases (IBDs) are regarded as disorders of unknown etiology with chronic relapsing inflammation affecting the gastrointestinal tract. Significant progress has been made in recent years toward understanding the pathogenesis of IBDs, although many aspects remain to be elucidated. Recent theories have emphasized the impact of genetic mutations in proteins linked to signaling pathways that influence the mechanisms of the innate and adaptive immune system. ${ }^{1-4}$

In this context, recent studies have drawn attention to the impact of the mitogen-activated protein kinase (MAPK) pathway on IBD. MAPKs include various subgroups such as p42/44 extracellular signaling kinase (ERK), p38, and c-Jun 
N-terminal kinase (JNK). Immune response mediators activate intracellular signaling cascades such as MAPK and initiate inflammatory responses. ${ }^{5}$ Increased expression and activation of MAPKs - and of JNK in particular - has been described in patients with IBD. ${ }^{6,7}$

Several studies have suggested that inhibition of JNK may alleviate intestinal inflammation. However, investigations of the effect of JNK on inflammation in intestinal inflammatory diseases have been limited due to a lack of specific inhibitors. ${ }^{8}$ Hollenbach et $\mathrm{al}^{9,10}$ reported a reduction in inflammatory activity when the nonspecific p38 MAPK inhibitor SB203580 was administered in colitis induced by DSS and 2,4,6-trinitrobenzenesulfonic acid (TNBS) colitis in mice. The stress-activated protein kinase inhibitor CNI-1493, a guanylhydrazone, has been found to be clinically beneficial in patients with Crohn's disease who did not respond to treatment with the anti-tumor necrosis factor- $\alpha$ (anti-TNF- $\alpha$ ) antibody infliximab. ${ }^{6}$ The compound SP600125 was the first specific inhibitor of JNK to be identified that shows activity against all isoforms of JNK. SP600125 is a reversible adenosine triphosphate-competitive inhibitor that interferes with the phosphorylation of c-Jun. Initial results for this substance showed that it inhibited activation and differentiation of $\mathrm{CD}^{+}{ }^{+}$cells. ${ }^{11}$ Assi et al ${ }^{12}$ found that inflammation was alleviated and TNF- $\alpha$ levels were reduced in a mouse model of acute DSS colitis. Interrelations with other pathways have also been observed at higher concentrations, however.

D-JNKI-1 is the first chemically synthesized JNK inhibitor that satisfies both of the important criteria for specific inhibition of JNK pathways: high specificity and membrane permeability. ${ }^{13,14} \mathrm{D}$-JNKI-1 inhibits phosphorylation of the JNK substrate c-Jun and is more potent than the small-molecule inhibitor SP600125 mentioned previously. ${ }^{15}$ D-JNKI-1 is produced by linking the 20 amino acid terminal JNK-inhibitory sequence (JNK binding domain) of JIP1/IB1 to a ten amino acid HIV-Tat transporter sequence for cell penetration. ${ }^{16-18}$ Peptidergic JNK inhibition using D-JNKI-1 has recently demonstrated preclinical and clinical benefit without undesirable side effects in several degenerative diseases such as traumatic hearing loss, ${ }^{17}$ colitis, ${ }^{19}$ uveitis,${ }^{20}$ diabetes,${ }^{21}$ myocardial ischemia, ${ }^{18}$ and ischemic stroke. $^{13,14,22}$

The present study investigated for the first time the therapeutic effect of the specific inhibiting peptide D-JNKI-1 on the clinical course, intestinal inflammation, and T-cell expression in a murine model of mild chronic DSS colitis.

\section{Materials and methods}

\section{Animals}

The experiments were conducted in female C57/BL6 mice (Charles River Ltd, Sulzfeld, Germany). The mice were all 10-12 weeks of age at the start of the experiment (weight $20-25 \mathrm{~g})$. They were housed five per cage in standard laboratory conditions (12-hour light/dark cycle, room temperature $24^{\circ} \mathrm{C}$, humidity $50 \%-60 \%$ ) and supplied with drinking water and food ad libitum (ssniff M-Z, $10 \mathrm{~mm}$, ssniff Spezialitäten, Soest, Germany). One group of mice $(n=5)$ served as controls and were given drinking water without any DSS.

\section{Induction of mild chronic DSS colitis}

Oral administration of DSS causes clinical, histological, and pathogenic features of T-helper (Th)1/Th2 chronic colitis resembling those of human $\mathrm{IBD}^{23}$ The DSS concentrations of $1.0 \%$ and $1.5 \%$ for inducing mild chronic colitis were established in previous studies (data not shown) and are suitable for experimental protocols.

The animals were randomized into two groups $(n=30)$. The mice received three cycles of treatment with DSS (molecular mass 36-44 kDa; MP Biomedicals, Aurora, OH, USA). The first group received 1.0\% DSS and the second group received $1.5 \%$ DSS for 5 days in demineralized drinking water. Each cycle of DSS treatment was followed by a 5-day interval with distilled water only. At the end of the third DSS cycle (after 30 days), the animals were sacrificed by carbon dioxide overdose.

\section{Administration of D-JNKI-I}

D-JNKI-1 (also termed XG-102) was synthesized and delivered by Xigen Ltd (Epalinges, Switzerland). D-JNKI-1 was dissolved in a $0.9 \%$ sodium chloride solution for subcutaneous application. Each group (the 1.0\% DSS group and the $1.5 \%$ DSS group) was randomly subdivided into an intervention group $(n=15)$ and a control group $(n=15)$. The mice in the intervention group received three subcutaneous nuchal administrations of $1 \mu \mathrm{g} / \mathrm{kg}$ D-JNKI-1 on days 2, 12 , and 22 . The mice in the control group received physiological saline (Braun, Melsungen, Germany) subcutaneously as a negative control at the same time points in a comparable stress situation.

\section{Assessment of disease activity}

Disease activity was quantified in all of the animals on a daily basis using the disease activity index (DAI), described elsewhere. ${ }^{24}$ The mice were weighed daily and visually inspected for stool consistency, diarrhea, and rectal bleeding. 
Hemoccult tests (Hemoccult; Sprothen Ltd, Stolberg, Germany) were performed daily to detect occult blood in the feces.

\section{Tissue samples}

After the mice had been sacrificed, the entire colon was removed. It was rinsed with normal saline (Braun) and divided into four anatomic parts - the cecum and proximal, medial, and distal colon. All of the dissection steps were performed on ice, and the tissue was snap-frozen in liquid nitrogen and stored at $-70^{\circ} \mathrm{C}$.

\section{Histological evaluation of inflammation}

For light microscopy, $5 \mu \mathrm{m}$ transverse cryostat sections from the four anatomic parts (cecum, proximal, medial, and distal colon) from each animal were cut on a rotary cryostat microtome (Leica CM1900; Leica Microsystems, Nussloch, Germany). Three consecutive sections per anatomic region and animal were mounted on coated glass slides and dried overnight at room temperature. The tissues were stained with hematoxylin and eosin (Vector Laboratories, Inc, Burlingame, CA, USA). Histological slides were examined under a light microscope (Leica DM4000 B microscope; Leica, Wetzlar, Germany). Mucosal damage in the entire colon was evaluated and graded independently by two blinded investigators (VB and IS). The degree of mucosal inflammation was graded semiquantitatively using the crypt damage score (CDS), as described previously. ${ }^{25}$ In addition, the score was slightly modified by taking immune cells in the mucosa and submucosa into account. With regard to intraobserver agreement, differences were noted in only $5 \%$ of the observations. Any differences in grading were resolved by a joint examination along with a third blinded examiner (SK).

\section{Immunofluorescence of activated lymphocytes}

Immunofluorescence analysis was carried out on $5 \mu \mathrm{m}$ transverse cryostat sections cut from the medial colon using a rotary cryostat microtome at $-18^{\circ} \mathrm{C}$ (Leica Microsystems). This location represents the segment of the colon with the highest inflammation. Four nonconsecutive sections were evaluated for each animal. The histological sections were mounted on poly-L-lysine slides (Super Starfrost, Menzel, Braunschweig, Germany), air-dried, and fixed in methanol at room temperature for 10 minutes. They were then blocked with Aurion BSA (Aurion BSA-c ${ }^{\mathrm{TM}}$; Aurion Immuno Reagents and Accessories, Wageningen, The Netherlands) for 45 minutes at room temperature.
The sections were subsequently incubated with the primary antibodies for 2 hours at room temperature: monoclonal rat anti-CD4 antibody $(31.25 \mu \mathrm{g} / \mathrm{mL}$; BD Biosciences, Heidelberg, Germany) diluted 1:200 in phosphatebuffered saline (PBS; Carl Roth, Karlsruhe, Germany) and monoclonal rat anti-CD 8 antibody $(0.2 \mathrm{mg} / \mathrm{mL}$; Abcam, Cambridge, UK) diluted 1:50 in PBS. After washing in PBS, tissue-bound antibodies were detected using monoclonal goat DyLight549 antibody $(0.5 \mathrm{mg} / \mathrm{mL}$; Jackson ImmunoResearch Laboratories, Inc, West Grove, PA, USA) diluted 1:200 in PBS.

Incubation with the secondary antibody was carried out for 2 hours at room temperature and then at $4{ }^{\circ} \mathrm{C}$ overnight. The sections were then washed in PBS. Each section had its own control using the secondary antibody only. Finally, the sections were mounted and analyzed using a fluorescence microscope (Leica DM4000 B microscope; Leica). Quantification of $\mathrm{CD}^{+}$and $\mathrm{CD} 8^{+}$cells was performed by examining two randomly selected high-power fields (with a magnification of $40,000 \times$ ).

\section{Statistical evaluation}

The results for the DAI and CDS were expressed as means \pm standard error of the mean (SEM). DAI values were analyzed using repeated-measures analysis of variance (ANOVA) over all time points. Comparison of CDS values between the groups was carried out using one-way ANOVA; paired comparisons were made using Tukey's test. The labeling density of $\mathrm{CD} 4^{+}$ and $\mathrm{CD} 8^{+}$cells was evaluated by $t$-test. $P$-values $\leq 0.05$ were considered statistically significant. Survival rates and hazard ratios were analyzed using a log-rank test and Cox regression analysis, with $P$-values $\leq 0.05$ considered statistically significant; $95 \%$ confidence intervals were also indicated.

\section{Animal care statement}

All of the animal experiments were performed in accordance with German legislation for the protection of animals and the National Institutes of Health guidelines for the use and care of laboratory animals. The experiments were all approved by the Ministry of Agriculture and the Environment (Ministerium fuer Landwirtschaft und Naturschutz), Kiel, Germany (V 312-72241.121-22 [60-6/08]).

\section{Results \\ Development of mild colitis using $1.0 \%$ and $1.5 \%$ DSS}

Untreated mice did not show any abnormal symptoms. In a control group of untreated mice $(n=5)$, the mean DAI ranged between 0 and 0.5 over all time points. 
All animals treated with DSS developed triphasic chronic colitis following cyclic administration of low-dose DSS (1.0\% and $1.5 \%)$, with moderate intensity measured on the DAI (Figure 1).

Previous studies by our group ${ }^{26-28}$ have already shown that during the first few days of DSS colitis, the animals respond to DSS treatment at different rates. This variability among the animals explains the lower DAI in the D-JNKI-1 group on day 1 (Figure 1). The parameters of stool consistency and rectal bleeding, which represent a very significant proportion of the DAI, already change after the first day of DSS treatment in some animals, whereas in other animals two or
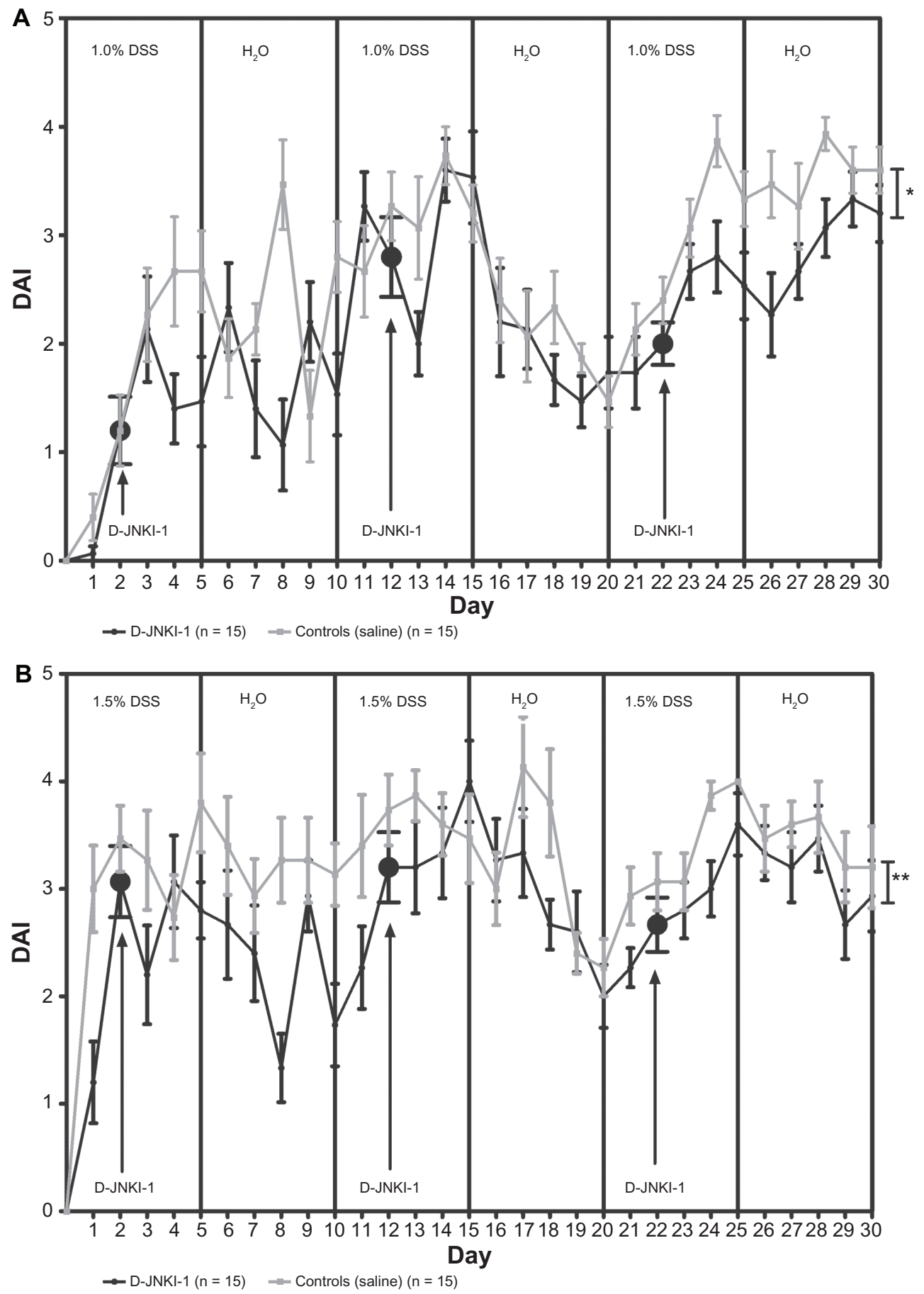

Figure I Time course of the disease activity index (DAI) (means \pm standard error of the mean) in the D-JNKI-I group and control group after administration of three cycles of $1.0 \%$ dextran sulfate sodium (DSS) (A) and I.5\% DSS (B).

Notes: Administration of D-JNKI-I on days 2, 12, and 22 resulted in clinical improvement, with significant differences in DAl scores between animals in the D-JNKI-I group and those in the control group ( $* P=0.013$ for $1.0 \%$ DSS and $* * P=0.007$ for $1.5 \%$ DSS; statistical evaluation: analysis of variance over all time points). 
three days of DSS treatment are necessary for colitis to start (Figures 2 and 3). During the subsequent course, all of the animals develop reproducible colitis.

In the experimental trial with $1.0 \%$ DSS, the DAI reached maximum scores after 14 days in the D-JNKI-1 group $(3.60 \pm 0.29)$ and after 28 days in the control group (DAI $3.93 \pm 0.15)$. Peak DAI values were detected at the end of each DSS treatment interval (Figure 1A). In the experimental trial with $1.5 \%$ DSS, the DAI reached maximum scores after 15 days in the D-JNKI-1 group (4.00 \pm 0.38$)$ and after 17 days in the control group (DAI $4.13 \pm 0.47$ ). Peak DAI values were detected at the end of each DSS treatment interval (Figure 1B). None of the animals in either experimental trial died (mortality $0 \%$ ). Subcutaneous injection of D-JNKI-1
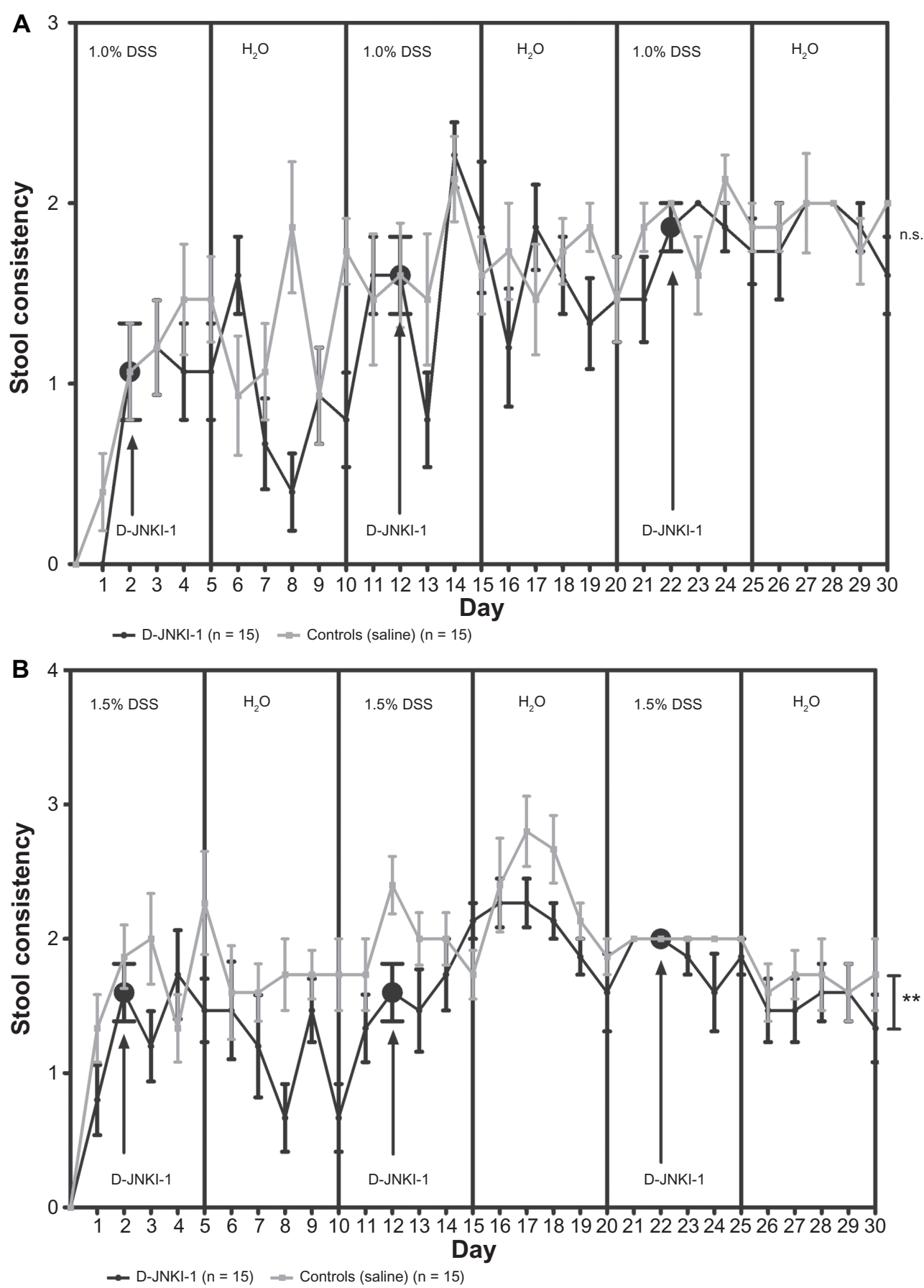

Figure 2 Time course of stool consistency (means \pm standard error of the mean) in the D-JNKI-I group and control group after administration of three cycles of I.0\% dextran sulfate sodium (DSS) (A) and I.5\% DSS (B).

Notes: Quantification of stool consistency: solid stool = 0 points; soft stool = 2 points; diarrhea $=4$ points. Administration of D-JNKI-I on days 2 , I2, and 22 in the I.5\% DSS group resulted in a significant difference in stool consistency $(* * P=0.006)$. In the $1.0 \%$ DSS group, only a trend toward a different stool consistency was demonstrated (not significant(n.s.); analysis of variance over all time points). 

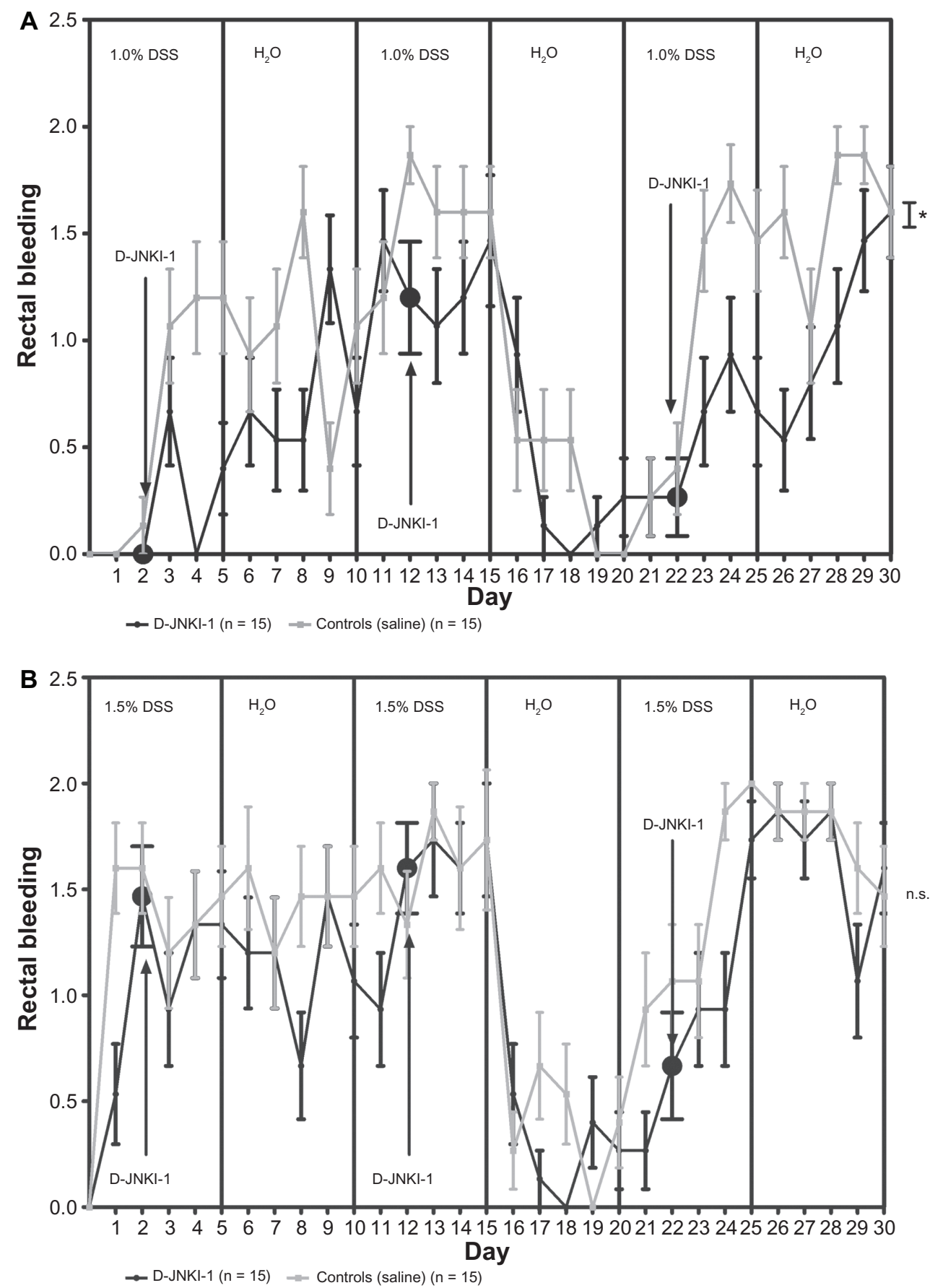

Figure 3 Time course of rectal bleeding (means \pm standard error of the mean) in the D-JNKI-I group and control group after administration of three cycles of I.0\% dextran sulfate sodium (DSS) (A) and I.5\% DSS (B).

Notes: Quantification of rectal bleeding: no blood = 0 points; occult blood = 2 points; macroscopic blood = 4 points. Administration of D-JNKI-I on days 2 , I2, and 22 in the I.0\% DSS group resulted in significantly $(* P=0.012)$ less rectal bleeding. In the I.5\% DSS group, only a trend toward less rectal bleeding was observed (not significant ( $\mathrm{n} . \mathrm{s}$.); analysis of variance over all time points).

significantly attenuated the clinical colitis parameters in both experimental trials. Differences in DAI values between the D-JNKI-1 and control groups were statistically significant, as evaluated using repeated-measures ANOVA over all time points $(1.0 \% \mathrm{DSS}, P=0.013 ; 1.5 \% \mathrm{DSS}, P=0.007$; D-JNKI-1 vs control group).
With regard to the DAI, D-JNKI-1 also tends to affect the parameters of stool consistency and rectal bleeding that compose the DAI. Significant results were observed in the $1.0 \%$ DSS group with regard to rectal bleeding $(P<0.05)$ and in the $1.5 \%$ DSS group with regard to stool consistency $(P<0.01)$ (Figures 2 and 3 ). 
No significant differences in body weight changes between the D-JNKI-1 groups and the control groups were observed after treatment with $1.0 \%$ and $1.5 \%$ DSS, probably because only mild colitis was induced.

\section{Histological scoring of the degree of inflammation}

DSS administration in both trial groups (1.0\% DSS and $1.5 \%$ DSS) caused moderate inflammation, characterized by infiltration of inflammatory cells and loss of goblet cells. As a further change characteristic of chronic inflammation, the submucosa was thickened, with pronounced edema. Tissue damage was scored by quantifying the length of crypts and lymphocyte infiltration.

After DSS treatment with 1.0\% DSS, histological scoring using the CDS developed by Egger et a ${ }^{25,29}$ showed increased crypt damage in the medial colon (CDS in the medial colon: D-JNKI-1 $8.53 \pm 0.82$ and saline $8.27 \pm 1.11$ ). However, these differences in CDS did not reach statistical significance (Figure 4A). Cyclic administration of 1.5\% DSS also led to the highest inflammation in the medial colon. Additionally, no significant differences were observed between the group treated with D-JNKI-1 and the control group (CDS in the medial colon: D-JNKI-1 $7.47 \pm 1.02$ and saline $7.93 \pm 0.77$ ) (Figures 4 and 5).

\section{Activated lymphocytes in the inflamed colon}

Immunofluorescence analysis of $\mathrm{CD} 4^{+}$and $\mathrm{CD} 8^{+}$cell expression was carried out in tissue from the medial colon in the $1.5 \%$ DSS experimental trial group, as this part of the colon developed the highest grade of inflammation. The total number of these cells was counted in two random sight fields. In both the D-JNKI-1 group and the control group, the number of $\mathrm{CD}^{+}$cells was lower than the total number of $\mathrm{CD} 8^{+}$ cells. After induction of chronic DSS colitis, CD8 staining was lower in the D-JNKI-1 group in comparison with the control group (15.8 \pm 1.55 and $20.72 \pm 1.81)$. CD4 staining was also lower in the D-JNKI-1 group than in the control group $(25.23 \pm 3.15$ and $28.60 \pm 3.29)$. However, neither the CD4 nor CD8 counts showed significant differences (Figures 6 and 7).

\section{Discussion}

In IBD, perpetual production of inflammatory mediators leads to inflammation of intestinal tissue. ${ }^{30-33}$ Although many forms of medical treatment have now been developed in order to control immune reactions and T-cell responses, relapsing
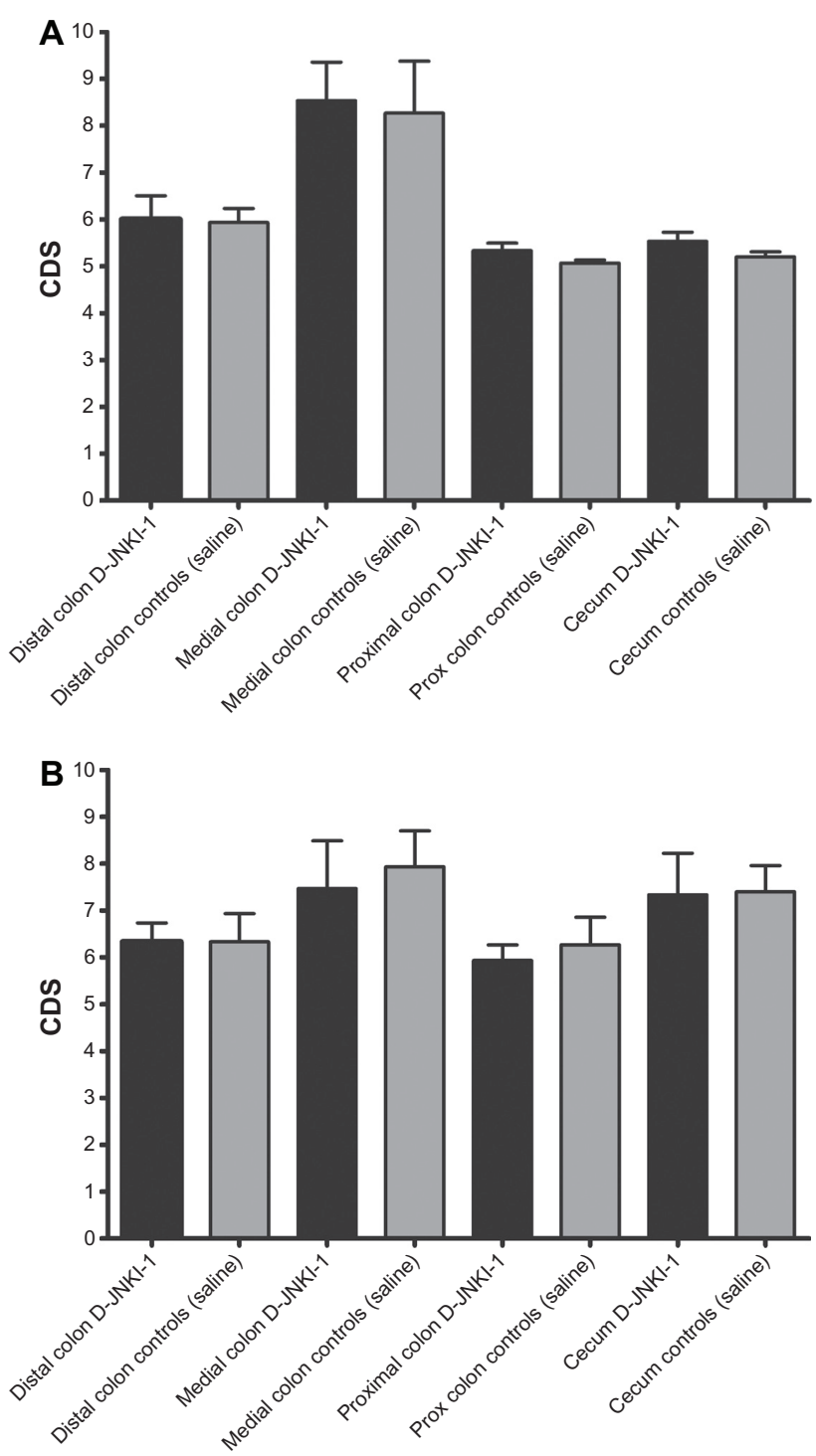

Figure 4 Histological evaluation of colitis using the crypt damage score (CDS) in the D-JNKI-I group and control group after administration of three cycles of $1.0 \%$ dextran sulfate sodium (DSS) (A) and I.5\% DSS (B) (means \pm standard error of the mean).

Notes: The most affected regions were the medial and distal colon. No differences between the two groups were observed after DSS treatment (CDS value comparison: one-way analysis of variance).

intestinal inflammation continues to be a major problem. New insights into the pathophysiology of IBD indicate that specific and targeted inhibition of inflammatory processes appears to be a promising treatment option. In this context, the modulation of cytokines such as interleukin (IL)-10, IL-11, and IL-12 has shown initially first positive effects on the progression of IBD. ${ }^{34-37}$ Treatment with infliximab, a monoclonal antibody against TNF- $\alpha$, is now a recognized and well-established therapy option in patients with severe courses of Crohn's disease. ${ }^{38,39}$ However, an important drawback in many current treatment options for IBD is the fact that the drug effect is often unspecific and associated with serious 

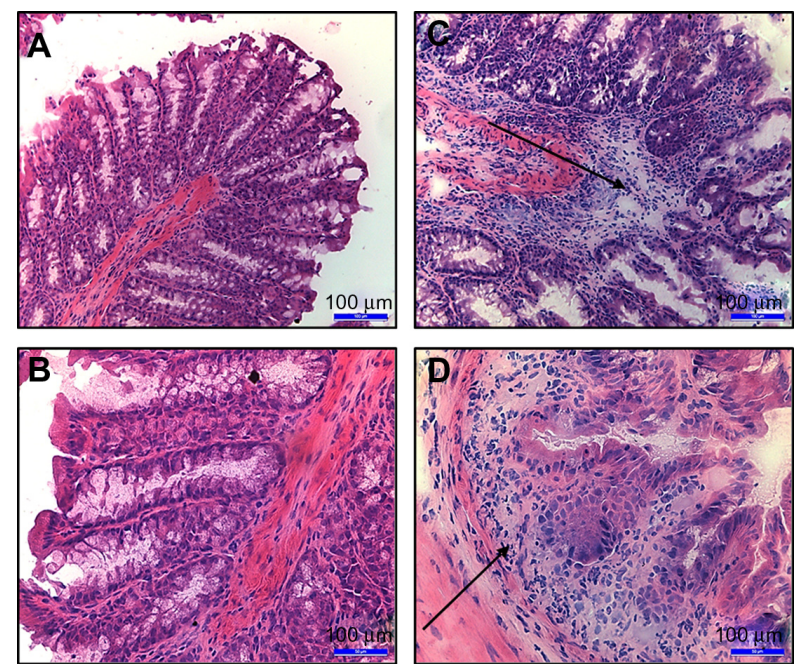

Figure 5 Representative hematoxylin-eosin stains from the medial colon in mice in the D-JNKI-I group and control group (I.5\% dextran sulfate sodium [DSS]). Normal mucosa with regular crypt architecture is seen in the D-JNKI-I group (A) magnification 20x and (B) magnification 40x. In contrast, mild changes in the crypt architecture were observed in the control group (C) magnification $20 \times$ and (D) magnification $40 \times$.

Note: The arrow indicates inflammatory infiltration.

adverse side effects. Further research is therefore necessary in order to optimize the treatment of patients with IBD.

In this context, the JNK pathway represents one possible target for intervening in the complex inflammatory mechanisms involved in IBD. As JNKs regulate various inflammatory reactions of the innate and specific immune response, promote inflammatory responses,${ }^{40}$ and control the expression of proinflammatory cytokines such as IL-2, IL-6, and TNF- $\alpha^{8}$ and the activation of inflammatory cells and apoptotic pathways, ${ }^{3,40,41}$ interventions in JNK signal transduction may provide new therapeutic opportunities for patients with IBD. However, the development of anti-JNK therapies has so far been limited due to a lack of

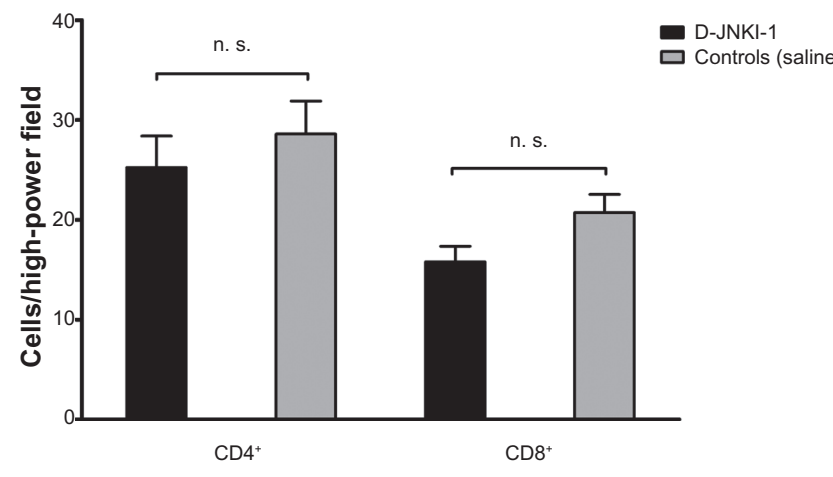

Figure 6 The labeling density of CD4 and CD8 (cells per high-power field) in the medial colon after administration of three cycles of $1.5 \%$ dextran sulfate sodium (DSS) in the D-JNKI-I group and control group.

Note: CD4 and CD8 staining was greater in the control group but did not show any significant differences (n.s.) in comparison with the D-JNKI-I group (statistical evaluation: $t$-test).
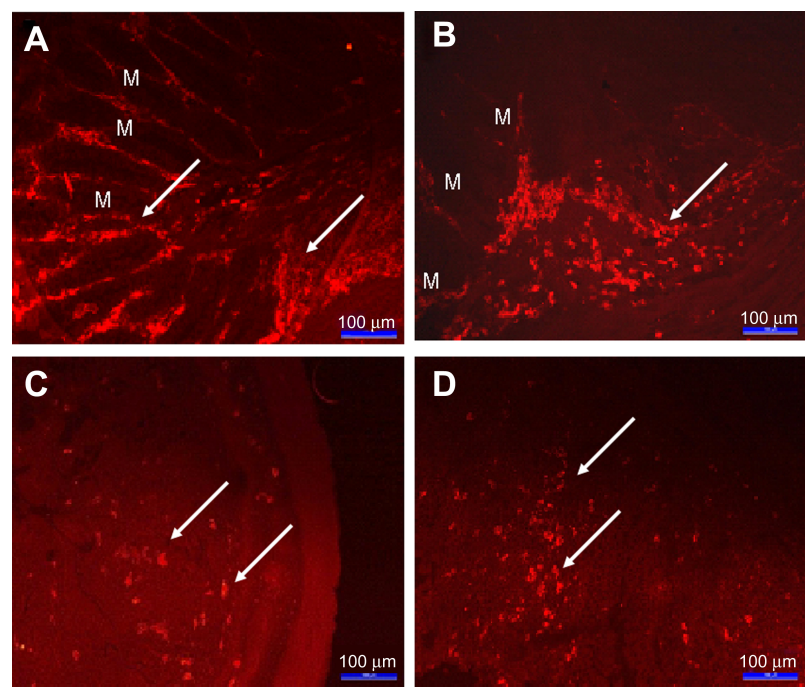

Figure 7 Immunofluorescence analysis (monoclonal goat DyLight549 antibody) of $\mathrm{CD}^{+}$and $\mathrm{CD}^{+}$cells in the medial colon after administration of three cycles of $1.5 \%$ dextran sulfate sodium (DSS) (magnification 20×). (A) $\mathrm{CD}^{+}$cells in the D-JNKI-I group. (B) CD4 $4^{+}$cells in the control group. (C) $\mathrm{CD}^{+}$cells in the D-JNKI-I group. (D) $\mathrm{CD}^{+}$cells in the control group.

Note: The arrow indicates inflammatory infiltration in the submucosa.

Abbreviation: M, mucosa.

appropriate inhibitors. Current JNK inhibitors provide poor specificity and cell permeability.

The present study investigated for the first time the effect of the highly specific JNK inhibitor D-JNKI-1 on mild chronic DSS colitis, a disease model with pathophysiological properties similar to those of ulcerative colitis. ${ }^{42}$ As a result of the toxic effect of the polysaccharide DSS on epithelial cells, the substance induces enteritis in the colon ${ }^{43}$ after oral administration, with clinically measurable symptoms such as weight loss and bloody diarrhea. ${ }^{44} \mathrm{At}$ an early stage, mucosal permeability is increased and the intestinal barrier function is destroyed. ${ }^{45}$ Intestinal antigens reach deeper layers of the intestinal wall and provoke an acute inflammatory response. In this model, the early inflammatory reaction is directed by macrophages and granulocytes. As the colitis continues, particularly in this model of chronic colitis, the influence of T lymphocytes in the Th1/Th2 cytokine profile increases. ${ }^{46,47}$ Further consequences include mucosal damage, which shows a linear relationship to the DSS concentration administered, with an increase in the proportion of typical Th1 cytokines: ie, TNF- $\alpha$, IL-12, and IL- $1 .{ }^{48}$ In addition, progressive shortening of the intestinal crypt structure leads to the development of inflammatory infiltration.

Two different intensities of chronic DSS colitis (1.0\% DSS and $1.5 \%$ DSS) were induced in the present study to analyze the influence of D-JNKI-1 on mild colitis. The clinical and histological features of inflammation were 
scored using the DAI and CDS, as described previously. ${ }^{25,29}$ In both experimental trials (1.0\% DSS and 1.5\% DSS), D-JNKI-1 clearly attenuated the disease activity and showed a therapeutic effect on the clinical course of DSS colitis. This observation is in line with recent studies in which less specific inhibitors of the MAPK/JNK pathway were used, such as SB203580 and CNI-1493.6,9,10

D-JNKI-1 also tends to affect the parameters of stool consistency and rectal bleeding that compose the DAI. Significant results were observed with regard to rectal bleeding in the $1.0 \%$ DSS group $(P<0.05)$ and with regard to stool consistency in the $1.5 \%$ DSS group $(P<0.01)$ (Figures 2 and 3 ). The reason for the lack of significance in the other groups and in relation to weight loss may be that only mild colitis was induced and only a few animals developed macroscopically apparent bloody diarrhea (four points in the DAI calculation). The majority of animals presented only with a smooth stool consistency (two points in the DAI for calculation).

Previous studies by our group demonstrated that cyclic administration of $1.0 \%$ DSS induces mild chronic colitis, whereas a further increase in the DSS dosage to $1.7 \%$ triggers severe colitis that is associated with high mortality rates $(>30 \%){ }^{26-28}$ Following this observation, the dosages of $1.0 \%$ DSS and $1.5 \%$ DSS were chosen in the present study to induce a mild form of chronic colitis. Histological analysis of the colon in the DSS-treated mice showed moderate inflammation, with slight immune cell infiltration and ulcerative tissue destruction. Unfortunately, no significant differences with regard to histological inflammation were seen after administration of D-JNKI-1. One possible reason for this might be the experimental protocol used, in which an apparently very mild form of chronic colitis was induced, which makes it difficult to assess the therapeutic effect of D-JNKI-1 on the histopathological structure of the colon mucosa. Prospective studies of severe colitis (with a DSS dosage of $1.7 \%$ or $2.0 \%$ ) and higher D-JNKI-1 dosage will be needed in order to investigate the therapeutic effect of D-JNKI-1 at the histological level.

In human IBD, pathological inflammation is associated with a proliferation of $\mathrm{CD}^{+}$and $\mathrm{CD}^{+}{ }^{+}$cells. ${ }^{49}$ It has been demonstrated that JNK1 and JNK2 play different roles during $\mathrm{CD}^{+}$and $\mathrm{CD} 4^{+}$cell activation. ${ }^{50}$ Perhaps again due to the only mild form of colitis induced, the present results did not reach statistical significance. Nevertheless, they show a trend toward reduced T-cell infiltration after administration of D-JNKI-1, particularly with $\mathrm{CD}^{+}$cells but also with $\mathrm{CD} 4^{+}$cells.

A comparable anti-inflammatory effect of D-JNKI-1 was previously described by our group ${ }^{19}$ in an acute form of severe TNBS colitis, which is an acute model of IBD with pathophysiological properties similar to those of Crohn's disease. ${ }^{51-53}$ Simultaneous subcutaneous application of D-JNKI-1 significantly reduced the DAI scores. After 72 hours - ie, at the end of the study - the colon was removed and analyzed immunohistochemically. D-JNKI-1 significantly reduced pathological changes such as ulceration and crypt deformation, immune cell pathology such as infiltration and the presence of $\mathrm{CD}^{+}$and $\mathrm{CD}^{+} 8^{+}$ cells, activation of caspase- 3 and expression, activation of the JNK substrate and transcription factor c-Jun, and production of TNF- $\alpha$. In TNBS colitis, a single administration of subcutaneous D-JNKI-1 was at least as effective as the daily oral administration of sulfasalazine used in the treatment of IBD.

With regard to the DSS model, previous studies by our group showed that JNK-dependent expression of the inflammatory cytokines TNF- $\alpha$, IL- 6 , and transforming growth factor- $\beta 1$ (TGFB1) apparently do not play a major role in the present model of mild chronic DSS colitis. In JNK1 knockout mice (MAPK8-/-), JNK2 knockout mice (MAPK9-/-), and wild-type controls (WT1, WT2), calibrator-normalized quantitative real-time polymerase chain reaction did not show any significant differences with regard to the expression of TNF- $\alpha$, TGFB1, or IL- $6 .{ }^{28}$ This finding is in line with observations by Alex et al, ${ }^{54}$ who reported an effect of these cytokines in acute DSS colitis but not in chronic DSS colitis. No cytokine analysis was therefore carried out in the present study.

Although there are no animal models that completely reflect all the pathophysiological aspects of human IBD, the successful alleviation of the clinical course of acute TNBS colitis and chronic DSS colitis in mice supports the hypothesis that inhibition of JNK may represent a promising therapeutic principle in IBD.

\section{Conclusion}

The present study shows that the JNK pathway represents a possible target for intervening in the complex inflammatory mechanisms involved in IBD. The therapeutic potential of the highly specific JNK inhibitor D-JNKI-1 was tested for the first time in a mild form of chronic DSS colitis in mice. It was found that in addition to being effective in the model of TNBS-induced colitis, ${ }^{19}$ D-JNKI-1 is also effective in another model of colitis, DSS-induced colitis. The results, showing an improvement in the clinical course, support the view that D-JNKI-1 has potential as a new form of treatment in patients with IBD. 


\section{Disclosure}

All authors declare no conflicts of interests. We do not have any paid or unpaid consulting, employment royalty, stock, patent agreement, position, or other financial relationship with any individual, company, or organization with a vested interest in the subject matter mentioned in the manuscript.

\section{References}

1. Watanabe T, Kitani A, Murray PJ, Strober W. NOD2 is a negative regulator of Toll-like receptor 2-mediated T helper type 1 responses. Nat Immunol. 2004;5(8):800-808.

2. Strober W, Murray PJ, Kitani A, Watanabe T. Signalling pathways and molecular interactions of NOD1 and NOD2. Nat Rev Immunol. 2006;6(1):9-20.

3. Dong C, Davis RJ, Flavell RA. MAP kinases in the immune response. Annu Rev Immunol. 2002;20:55-72.

4. Sumbayev VV, Yasinska IM. Role of MAP kinase-dependent apoptotic pathway in innate immune responses and viral infection. Scand $J$ Immunol. 2006;63(6):391-400.

5. Hommes DW, Peppelenbosch MP, van Deventer SJ. Mitogen activated protein (MAP) kinase signal transduction pathways and novel antiinflammatory targets. Gut. 2003;52(1):144-151.

6. Hommes D, van den Blink B, Plasse T, et al. Inhibition of stressactivated MAP kinases induces clinical improvement in moderate to severe Crohn's disease. Gastroenterology. 2002;122(1):7-14.

7. Waetzig GH, Seegert D, Rosenstiel P, Nikolaus S, Schreiber S. p38 mitogen-activated protein kinase is activated and linked to TNF-alpha signaling in inflammatory bowel disease. J Immunol. 2002;168(10):5342-5351.

8. Roy PK, Rashid F, Bragg J, Ibdah JA. Role of the JNK signal transduction pathway in inflammatory bowel disease. World $J$ Gastroenterol. 2008;14(2):200-202.

9. Hollenbach E, Neumann M, Vieth M, Roessner A, Malfertheiner P, Naumann M. Inhibition of p38 MAP kinase- and RICK/NF-kappaBsignaling suppresses inflammatory bowel disease. FASEB J. 2004; 18(13):1550-1552.

10. Hollenbach E, Vieth M, Roessner A, Neumann M, Malfertheiner P, Naumann M. Inhibition of RICK/nuclear factor-kappaB and p38 signaling attenuates the inflammatory response in a murine model of Crohn disease. J Biol Chem. 2005;280(15):14981-14988.

11. Bennett BL, Sasaki DT, Murray BW, et al. SP600125, an anthrapyrazolone inhibitor of Jun N-terminal kinase. Proc Natl Acad Sci U S A. 2001;98(24):13681-13686.

12. Assi K, Pillai R, Gomez-Munoz A, Owen D, Salh B. The specific JNK inhibitor SP600125 targets tumour necrosis factor-alpha production and epithelial cell apoptosis in acute murine colitis. Immunology. 2006; 118(1):112-121.

13. Wiegler K, Bonny C, Coquoz D, Hirt L. The JNK inhibitor XG-102 protects from ischemic damage with delayed intravenous administration also in the presence of recombinant tissue plasminogen activator. Cerebrovasc Dis. 2008;26(4):360-366.

14. Borsello T, Clarke PG, Hirt L, et al. A peptide inhibitor of c-Jun $\mathrm{N}$-terminal kinase protects against excitotoxicity and cerebral ischemia. Nat Med. 2003;9(9):1180-1186.

15. Zhuang ZY, Wen YR, Zhang DR, et al. A peptide c-Jun N-terminal kinase (JNK) inhibitor blocks mechanical allodynia after spinal nerve ligation: respective roles of JNK activation in primary sensory neurons and spinal astrocytes for neuropathic pain development and maintenance. J Neurosci. 2006;26(13):3551-3560.

16. Vives E, Brodin P, Lebleu B. A truncated HIV-1 Tat protein basic domain rapidly translocates through the plasma membrane and accumulates in the cell nucleus. J Biol Chem. 1997;272(25): $16010-16017$
17. Wang J, Van De Water TR, Bonny C, de Ribaupierre F, Puel JL, Zine A. A peptide inhibitor of c-Jun N-terminal kinase protects against both aminoglycoside and acoustic trauma-induced auditory hair cell death and hearing loss. J Neurosci. 2003;23(24):8596-8607.

18. Milano G, Morel S, Bonny C, et al. A peptide inhibitor of c-Jun $\mathrm{NH} 2$-terminal kinase reduces myocardial ischemia-reperfusion injury and infarct size in vivo. Am J Physiol Heart Circ Physiol. 2007;292(4): H1828-H1835.

19. Reinecke K, Eminel S, Dierck F, et al. The JNK Inhibitor XG-102 Protects against TNBS-induced colitis. PLoS One. 2012;7(3):e30985.

20. Touchard E, Omri S, Naud MC, et al. A peptide inhibitor of c-Jun $\mathrm{N}$-terminal kinase for the treatment of endotoxin-induced uveitis. Invest Ophthalmol Vis Sci. 2010;51(9):4683-4693.

21. Bonny C, Oberson A, Negri S, Sauser C, Schorderet DF. Cell-permeable peptide inhibitors of JNK: novel blockers of beta-cell death. Diabetes. 2001;50(1):77-82.

22. Benakis C, Bonny C, Hirt L. JNK inhibition and inflammation after cerebral ischemia. Brain Behav Immun. 2010;24(5):800-811.

23. Strober W, Fuss IJ, Blumberg RS. The immunology of mucosal models of inflammation. Annu Rev Immunol. 2002;20:495-549.

24. Cooper HS, Murthy SN, Shah RS, Sedergran DJ. Clinicopathologic study of dextran sulfate sodium experimental murine colitis. Lab Invest. 1993;69(2):238-249.

25. Egger B, Procaccino F, Lakshmanan J, et al. Mice lacking transforming growth factor alpha have an increased susceptibility to dextran sulfateinduced colitis. Gastroenterology. 1997;113(3):825-832.

26. Chromik AM, Muller AM, Albrecht M, et al. Oral administration of taurolidine ameliorates chronic DSS colitis in mice. J Invest Surg. 2007;20(5):273-282.

27. Chromik AM, Muller AM, Korner J, et al. Genetic deletion of JNK1 and JNK2 aggravates the DSS-induced colitis in mice. J Invest Surg. 2007;20(1):23-33.

28. Kersting S, Reinecke K, Hilgert C, et al. Knockout of the c-Jun N-terminal kinase 2 (JNK2) aggravates the development of mild chronic dextran sulfate sodium colitis independently of expression of the intestinal cytokines TNFo, TGFB1 and IL-6. J Inflamm Res. 2013 (Accepted for publication).

29. Egger B, Carey HV, Procaccino F, et al. Reduced susceptibility of mice overexpressing transforming growth factor alpha to dextran sodium sulphate induced colitis. Gut. 1998;43(1):64-70.

30. Pizarro TT, Cominelli F. Cytokine therapy for Crohn's disease: advances in translational research. Annu Rev Med. 2007;58: 433-444.

31. Broom OJ, Widjaya B, Troelsen J, Olsen J, Nielsen OH. Mitogen activated protein kinases: a role in inflammatory bowel disease? Clin Exp Immunol. 2009;158(3):272-280.

32. van Montfrans C, Peppelenbosch M, te Velde AA, van Deventer S. Inflammatory signal transduction in Crohn's disease and novel therapeutic approaches. Biochem Pharmacol. 2002;64(5-6):789-795.

33. Rogler G. Update in inflammatory bowel disease pathogenesis. Curr Opin Gastroenterol. 2004;20(4):311-317.

34. Mannon PJ, Fuss IJ, Mayer L, et al. Anti-interleukin-12 antibody for active Crohn's disease. N Engl J Med. 2004;351(20):2069-2079.

35. Sands BE, Bank S, Sninsky CA, et al. Preliminary evaluation of safety and activity of recombinant human interleukin 11 in patients with active Crohn's disease. Gastroenterology. 1999;117(1):58-64.

36. Sands BE, Winston BD, Salzberg B, et al. Randomized, controlled trial of recombinant human interleukin-11 in patients with active Crohn's disease. Aliment Pharmacol Ther. 2002;16(3):399-406.

37. Schreiber S, Fedorak RN, Nielsen $\mathrm{OH}$, et al. Safety and efficacy of recombinant human interleukin 10 in chronic active Crohn's disease. Crohn's Disease IL-10 Cooperative Study Group. Gastroenterology. 2000;119(6):1461-1472.

38. Present DH, Rutgeerts $\mathrm{P}$, Targan $\mathrm{S}$, et al. Infliximab for the treatment of fistulas in patients with Crohn's disease. $N$ Engl J Med. 1999;340(18): 1398-1405. 
39. Rutgeerts P, D'Haens G, Targan S, et al. Efficacy and safety of retreatment with anti-tumor necrosis factor antibody (infliximab) to maintain remission in Crohn's disease. Gastroenterology. 1999;117(4): 761-769.

40. Davis RJ. Signal transduction by the JNK group of MAP kinases. Cell. 2000;103(2):239-252.

41. Kyriakis JM, Avruch J. Mammalian mitogen-activated protein kinase signal transduction pathways activated by stress and inflammation. Physiol Rev. 2001;81(2):807-869.

42. Okayasu I, Hatakeyama S, Yamada M, Ohkusa T, Inagaki Y, Nakaya R. A novel method in the induction of reliable experimental acute and chronic ulcerative colitis in mice. Gastroenterology. 1990;98(3): 694-702.

43. Dieleman LA, Ridwan BU, Tennyson GS, Beagley KW, Bucy RP, Elson CO. Dextran sulfate sodium-induced colitis occurs in severe combined immunodeficient mice. Gastroenterology. 1994;107(6): $1643-1652$.

44. Ott C, Obermeier F, Thieler S, et al. The incidence of inflammatory bowel disease in a rural region of Southern Germany: a prospective population-based study. Eur J Gastroenterol Hepatol. 2008;20(9): 917-923.

45. Kitajima S, Takuma S, Morimoto M. Changes in colonic mucosal permeability in mouse colitis induced with dextran sulfate sodium. Exp Anim. 1999;48(3):137-143.

46. Dieleman LA, Palmen MJ, Akol H, et al. Chronic experimental colitis induced by dextran sulphate sodium (DSS) is characterized by Th1 and Th2 cytokines. Clin Exp Immunol. 1998;114(3): 385-391.
47. Shintani N, Nakajima T, Okamoto T, Kondo T, Nakamura N, Mayumi T. Involvement of $\mathrm{CD}^{+} \mathrm{T}$ cells in the development of dextran sulfate sodium-induced experimental colitis and suppressive effect of $\mathrm{IgG}$ on their action. Gen Pharmacol. 1998;31(3):477-481.

48. Egger B, Bajaj-Elliott M, MacDonald TT, Inglin R, Eysselein VE, Buchler MW. Characterisation of acute murine dextran sodium sulphate colitis: cytokine profile and dose dependency. Digestion. 2000;62(4): 240-248.

49. Duchmann R, Kaiser I, Hermann E, Mayet W, Ewe K, Meyer zum Buschenfelde $\mathrm{KH}$. Tolerance exists towards resident intestinal flora but is broken in active inflammatory bowel disease (IBD). Clin Exp Immunol. 1995;102(3):448-455.

50. Conze D, Krahl T, Kennedy N, et al. c-Jun NH(2)-terminal kinase (JNK) 1 and JNK2 have distinct roles in CD8(+) T cell activation. J Exp Med. 2002;195(7):811-823.

51. te Velde AA, Verstege MI, Hommes DW. Critical appraisal of the current practice in murine TNBS-induced colitis. Inflamm Bowel Dis. 2006;12(10):995-999.

52. Wirtz S, Neufert C, Weigmann B, Neurath MF. Chemically induced mouse models of intestinal inflammation. Nat Protoc. 2007;2(3):541-546.

53. Carvalho AT, Souza H, Carneiro AJ, et al. Therapeutic and prophylactic thalidomide in TNBS-induced colitis: synergistic effects on TNF-alpha, IL-12 and VEGF production. World J Gastroenterol. 2007;13(15) 2166-2173.

54. Alex P, Zachos NC, Nguyen T, et al. Distinct cytokine patterns identified from multiplex profiles of murine DSS and TNBS-induced colitis. Inflamm Bowel Dis. 2009;15(3):341-352.
Journal of Inflammation Research

\section{Publish your work in this journal}

The Journal of Inflammation Research is an international, peer-reviewed open-access journal that welcomes laboratory and clinical findings on the molecular basis, cell biology and pharmacology of inflammation including original research, reviews, symposium reports, hypothesis formation and commentaries on: acute/chronic inflammation; mediators of inflamma-

\section{Dovepress}

tion; cellular processes; molecular mechanisms; pharmacology and novel anti-inflammatory drugs; clinical conditions involving inflammation. The manuscript management system is completely online and includes a very quick and fair peer-review system. Visit http://www.dovepress.com/ testimonials.php to read real quotes from published authors. 